\title{
ANÁLISE DO SISTEMA PROGNÓSTICO DE MORTALIDADE APACHE II EM PACIENTES CIRÚRGICOS DE UNIDADE DE TERAPIA INTENSIVA
}

\author{
MORTALITY EVALUATED BY APACHE II PROGNOSTIC SYSTEM IN A \\ SURGICAL CRITICAL CARE UNIT
}

\author{
Waldiere Machado Gonçalves, $\mathrm{RCBC}^{-S C} \mathrm{C}^{1}$ \\ Nicolau Fernandes Kruel, TCBC-SC ${ }^{2}$ \\ Pedro de Almeida Araújo, TCBC-SC ${ }^{3}$ \\ Diana Oliveira Teixeira ${ }^{4}$
}

\begin{abstract}
RESUMO: O presente trabalho foi realizado na Unidade de Terapia Intensiva (UTI) do Hospital Regional de São José Dr. Homero de Miranda Gomes (HRSJHMG) com o objetivo de observar a aplicabilidade e a exatidão do sistema preditivo Acute Phisiology and Chronic Health Evaluatiaon II (APACHE II) nos pacientes cirúrgicos desta unidade, comparar os resultados obtidos com aqueles observados em outros centros, e, talvez, como principal objetivo, servir como um parâmetro basal para a performance desta UTI, no que refere à abordagem/tratamento do paciente cirúrgico. Trata-se de um estudo clínico prospectivo, não controlado, realizado na UTI do HRSJHMG. Foram estudados consecutivamente todos os pacientes cirúrgicos que necessitaram de cuidados intensivos por um período superior a 24 horas no período de janeiro a julho de 1996. Os principais resultados obtidos demonstraram haver uma boa correlação entre a mortalidade real $(38,09 \%)$ e a prevista pelo sistema APACHE II (24,57\%), apresentando uma relação entre a mortalidade real e a prevista (SRM) de 1,55.
\end{abstract}

Unitermos: APACHE II; Preditores de sobrevida.

\section{INTRODUÇÃO}

O exercício da medicina permanece ainda como um processo largamente empírico. A despeito da concentração de recursos materiais e humanos cada vez maiores, que se exigem para a adequada abordagem terapêutica, de suporte ou de monitoração do paciente agudamente enfermo, isto nem sempre se acompanha, como se esperaria, de melhores resultados em termos de sobrevida e qualidade de vida.

Objetivando uma melhor utilização dos recursos destinados à medicina intensiva, procurou-se estabelecer parâmetros para estratificar os pacientes que estão usufruindo destes recursos e se realmente estes se beneficiarão da utilização dos mesmos. Com base nestas preocupações, nos últimos 15 anos uma série de importantes estudos foi realizada visando o desenvolvimento de índices prognósticos para avaliação de pacientes gravemente enfermos internados em UTIs. ${ }^{1-5} \mathrm{Os}$ objetivos principais destes sistemas prognósticos são:

a) estratificação de risco, permitindo agrupar pacientes de acordo com a gravidade da doença de base e os distúrbios fisiológicos agudos; b) homogeneizar amostras em estudos multicêntricos, para saber se há similaridade entre as populações em questão; c) avaliar a performance das condutas empregadas nas UTIs (não do ponto de vista individual) e, d) comparar o desempenho entre diferentes UTIs. ${ }^{6}$

O primeiro índice prognóstico de avaliação de gravidade da doença, o Acute Phisiology and Chronic Health Evaluation (APACHE), foi introduzido por Knaus et al, em 1981. ${ }^{3}$ Este, por sua vez, foi precedido pelo desenvolvimento de índices prognósticos dirigidos para doenças específicas, como o Glasgow coma score para pacientes com doença neurológica, a classificação de Killip para o infarto agudo do miocárdio, e

1. Médicos Residentes de Cirurgia Geral do HRSJHMG.

2. Chefe do Serviço de Cirurgia Geral do HRSJHMG.

3. Coordenador da Residência em Cirurgia Geral do HRSJHMG.

4. Acadêmica de Medicina da UFSC.

Recebido em 21/5/97

Aceito para publicação em 11/1/99

Trabalho realizado no Serviço de Cirurgia Geral do Hospital Regioal de São José - Dr. Homero de Miranda Gomes - HRSJHMG. 
a classificação de Ransom para pancreatite. A base para o desenvolvimento do sistema APACHE estava na hipótese de que a gravidade da doença aguda poderia ser mensurada através da quantificação do grau de anormalidades das múltiplas variáveis fisiológicas. Contudo, o APACHE original é um sistema complexo, com muitas variáveis e de difícil aplicação, inferindo a necessidade de um modelo mais simplificado. Desta necessidade e de esforços continuados, surgiu o sistema APACHE II, uma versão modificada e aprimorada do original, desenvolvida pelo mesmo grupo de estudo, baseada num banco de dados com mais de 5 mil pacientes. Esta nova versão, mais acurada quanto ao poder preditivo e de uso clínico mais simples, foi apresentada à comunidade científica em $1985 .{ }^{4}$ O número de medidas fisiológicas foi reduzido de 34 para apenas 12. Em razão da idade e dos problemas crônicos graves de saúde de procedência imediata reduzirem a reserva fisiológica ao stress, estes ganharam destaque na versão APACHE II. ${ }^{4}$

Continuando sua linha de pesquisa como membro da ICU Research Unit, George Washington School of Medicine, Washington D.C., Knaus et al vieram a desenvolver e publicar mais recentemente o Acute Physiology and Chronic Health Evaluation III (APACHE III). ${ }^{5}$

Em nosso meio, a situação não é diferente: a dificuldade para obtenção de recursos, e a angústia e a incerteza do real benefício que estes recursos estão trazendo ao paciente, nos motivaram a realização deste trabalho, um trabalho pioneiro realizado na UTI do HRSJ, que tem como objetivo observar a aplicabilidade e a acurácia do sistema preditivo APACHE II nos pacientes cirúrgicos desta unidade, comparar os resultados obtidos com aqueles observados em outros centros, e, talvez, como principal objetivo, servir como um parâmetro basal para a performance desta UTI, no que tange à abordagem/tratamento do paciente cirúrgico. Cabe ressaltar que este trabalho não tem o objetivo de tornar-se instrumento para amparar decisões individuais. O sistema a ser estudado será Acute Physiology and Chronic Health Evaluation II(APACHE II),${ }^{4}$ uma evolução do seu precursor o sistema APACHE,${ }^{3}$ devido a sua maior aplicabilidade.

\section{PACIENTES E MÉTODOS}

Trata-se de um estudo clínico prospectivo, realizado na UTI do HRSJHMG, uma unidade mista de internação com cinco leitos. Foram estudados consecutivamente todos os pacientes cirúrgicos que necessitaram de cuidados intensivos por um período superior a 24 horas.

O sistema preditivo de mortalidade intra-hospitalar para pacientes severamente enfermos desenvolvido por Willians Knaus em 1985, o Acute Phisiology and Cronic Health Evaluation II (APACHE II), foi utilizado como descrito por Knaus nos pacientes cirurgicos internados na UTI deste hospital, com exceção à escala de coma de Glasgow, que utilizou a melhor pontuação das primeiras 24 horas; todas as demais variáveis serão consideradas para cálculo do APS as piores variáveis das primeiras 24 horas. Para colheita dos dados utilizou-se um formulário padrão, e, com os dados em mãos das variáveis fisiológicas das primeiras 24 horas, obtevese $o$ acute score phisiology ou escore fisiológico agudo; além deste, o estado prévio de saúde e a procedência imediata também foram avaliados.

A pontuação máxima para o score APACHE II é de 71 pontos, assim obtidos: idade, máximo de 6 pontos; APS, máximo de 60 pontos; e avaliação do estado prévio de saúde e procedência imediata, máximo de 5 pontos (pacientes nãocirúrgicos ou submetidos a cirurgia de urgência com disfunção orgânica grave prévia recebiam 5 pontos, pacientes submetidos à cirurgia eletiva recebiam 2 pontos).

A equação final para cálculo do índice APACHE II é expressa: In (R/1-R) $=-3,517+($ APACHE II SCORE x 0,146) $+(0,603$ somente se cirurgia de emergência $)+$ (Diagnóstico específico).

O índice APACHE II expressa o valor preditivo de mortalidade em termos percentuais, de modo que um índice APACHE II de 50 pontos percentuais reflete o prognóstico de mortalidade intra-hospitalar de 50\%.

Os dados foram armazenados em um banco de dados (Microsoft Excel 5.0) para análise estatística. Utilizou-se média e desvio padrão para expressar as variáveis numéricas contínuas. O teste de t-student será utilizado para comparar variáveis não binominais e numéricas e o teste das proporções (qui-quadrado) para as variáveis binominais, com correção de Yates para pequenas amostras.

\section{RESULTADOS}

De janeiro a julho de 1996, foram internados na Unidade de Terapia Intensiva do Hospital Regional de São José - Dr. Homero de Miranda Gomes, 84 pacientes cirúrgicos que necessitaram de cuidados intensivos por um período superior a 24 horas. A faixa etária predominante da amostra ficou entre 56 e 65 anos (Figura 1). A média etária dos pacientes ficou em 52,62 $\pm 19,75$ anos, e variou de 13 a 86 anos. Quanto ao sexo, 61 pacientes eram do sexo masculino $(72,62 \%)$ e 23 do sexo feminino $(27,38 \%)$ (Figura 2).

Quanto à procedência, 56 pacientes $(66,67 \%)$ foram submetidos a cirurgia de urgência e $28(33,33 \%)$ a cirurgia eletiva. A taxa de mortalidade foi de $38,09 \%$ (32 pacientes).

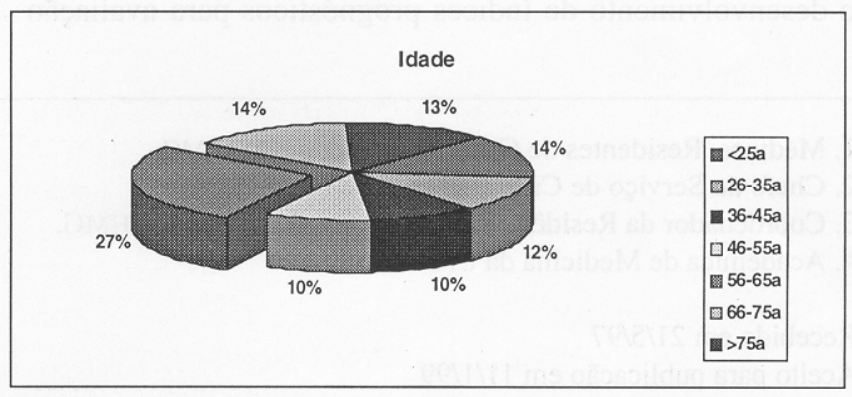

Figura 1 - Distribuição dos pacientes segundo a faixa etária 


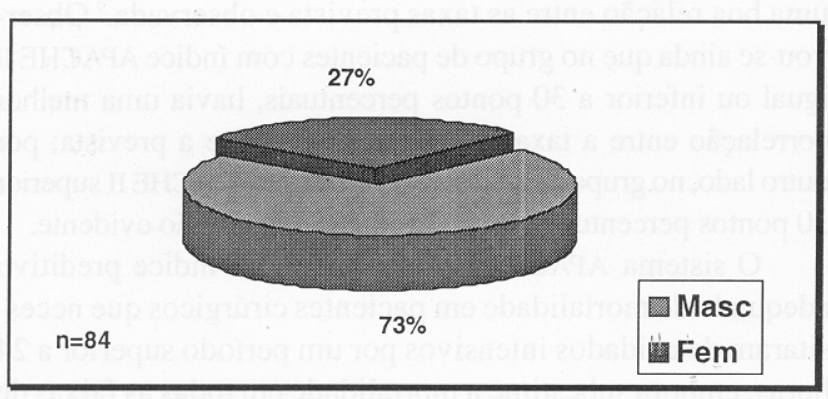

Figura 2 - Distribuição dos pacientes quanto ao sexo

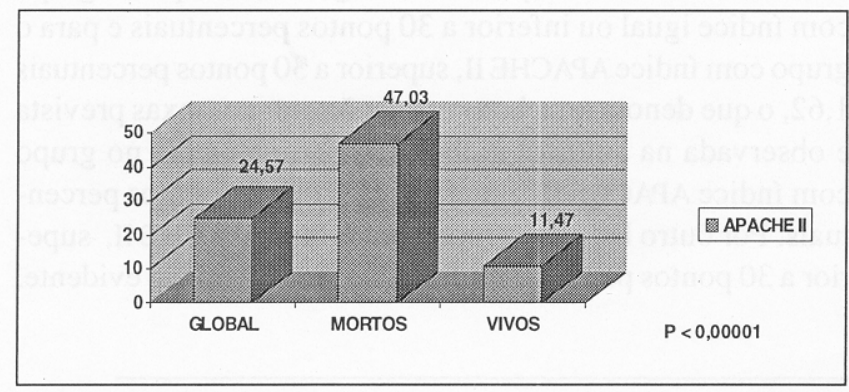

Figura 3 - Apresentação do índice APACHE II médio para amostra global, para o grupo dos não-sobreviventes e para o grupo dos sobreviventes

Em relação ao sistema APACHE II, observou-se que o score médio da amostra global foi de 17,06 $\pm 9,37$; no grupo dos sobreviventes o score médio foi de 12,31 , enquanto o dos não-sobreviventes foi de 24,78 ( $\mathrm{p}<0,0003)$. O índice APACHE II da amostra total alcançou uma média dę 24,57 \pm 2 8,80; no grupo dos sobreviventes ficou em 11,47 e no grupo dos não- sobreviventes 47,03 ( $\mathrm{p}<0,00001$ ) (Figura 3).

O sistema APACHE II imputa determinado score dependendo dos valores da variáveis fisiológicas. Quanto se analisou a distribuição destes scores na amostra, observamos uma grande variabilidade. A menor faixa de score (entre 0 e 5,56) continha um paciente $(1,19 \%)$, e a maior (acima de 37,4$)$, um paciente $(1,19 \%)$. No intervalo entre estes extremos, dividimos mais oito grupos com variação média de 4,5 pontos. $\mathrm{O}$ intervalo predominante foi entre 10,1 e 14,7 com 21 pacientes $(25,0 \%)$. O score médio foi de 17,06 $\pm 9,37$ pontos (Figura 4).

$\mathrm{O}$ método de validação prescreve que o índice APACHE II de mortalidade intra-hospitalar deve ser semelhante, do ponto de vista estatístico, ao índice real de mortalidade observado ao final do estudo

Ao se analisar o índice APACHE II, dividindo-o por faixa de variações de 10 pontos percentuais, observou-se que nas faixas de variações abaixo de 30 pontos percentuais houve uma boa correlação entre o índice APACHE II e a mortalidade real (Figura 5).

Procurou-se separar os pacientes em dois grupos: com índice abaixo e acima de 30 pontos percentuais respectivamente. Foi calculada a Standardized Mortality Rate (SMR)

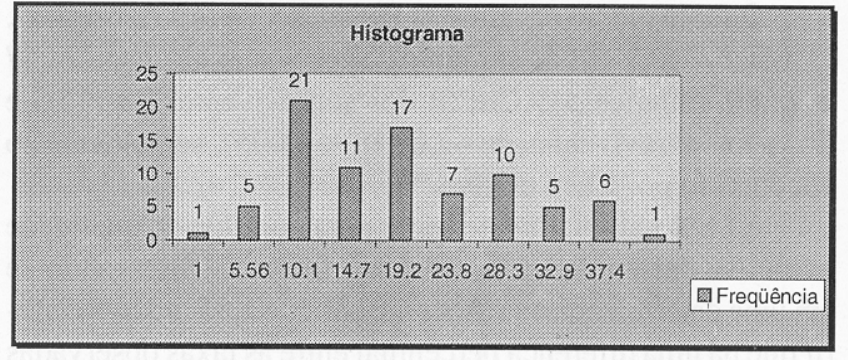

Figura 4-Distribuição dos pacientes segundo o score APACHE II

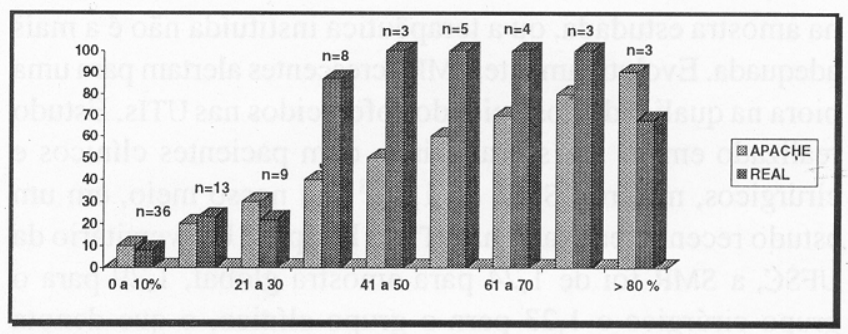

Figura 5-Relação entre mortalidade prevista pelo índice APACHE II e mortalidade real

(razão da mortalidade observada pela padrão) para os diversos grupos. Para a amostra global, o valor encontrado foi de 1,55; para o grupo com índice APACHE II igual ou inferior a 30 pontos percentuais foi de 1,36 e para o grupo com índice acima de 30 pontos percentuais, 1,62.

\section{DISCUSSÃO}

O APACHE II tem sido validado extensivamente por estudos nos diversos países, ${ }^{4,7}$ inclusive no Brasil. ${ }^{8,9}$ Em um estudo envolvendo 10 UTIs brasileiras caracterizou-se uma população com índices de gravidade superior ao observado no estudo original por Knaus. O reflexo desta maior gravidade resultou numa razão entre a mortalidade real e a prevista de $1,66 .^{8}$

O sistema APACHE II tem se mostrado um eficiente modelo preditivo de mortalidade intra-hospitalar para grupos de pacientes não selecionados em países industrializados ${ }^{7} \mathrm{ou}$ subdesenvolvidos. ${ }^{9}$ Alguns estudos demonstraram que o APACHE II perde seu poder preditivo quando da utilização de critérios específicos de seleção de pacientes. ${ }^{5}$

$\mathrm{Na}$ amostra estudada, a faixa predominante se situou entre 56 e 65 anos (27,0\%), o que merece ser ressaltado, pois a participação desta variável na composição da equação APACHE II, é considerada. A idade média foi de 52,65 $\pm 19,75$ anos. Em estudos envolvendo o mesmo grupo de pacientes, Silva encontrou uma média etária de aproximadamente 53,92 anos respectivamente. ${ }^{9}$ Pode-se observar que, em estudos semelhantes, a média etária ficou em torno de 54 anos. ${ }^{7,10}$

A taxa de mortalidade geral foi de $38,09 \%$. Em estudos envolvendo pacientes cirúrgicos admitidos em UTIs, encontraram-se taxas de mortalidade que variaram de $17 \%$ a $26 \% .^{9,11}$ 
O score médio da amostra global ficou em 17,06 $\pm 9,37$. O índice APACHE II do conjunto dos pacientes alcançou 24,57 $\pm 28,80$, o índice APACHE II dos sobreviventes de 11,03 e dos não-sobreviventes de 47,03 ( $p<0,005$ ), semelhante ao encontrado por Chen e Silva. ${ }^{11}$

A SMR habitualmente serve para controle de qualidade evolutiva das unidades de terapia intensiva. Observando-se em uma determinada amostra uma SMR de 1,55, pode-se inferir que há uma diferença percentual entre as taxas observadas e previstas $(55 \%)$. Deste dado, pode-se subtrair duas hipóteses; ou o sistema APACHE II subestima a taxa de mortalidade na amostra estudada, ou a terapêutica instituída não é a mais adequada. Evolutivamente, SMRs crescentes alertam para uma piora na qualidade dos cuidados oferecidos nas UTIs. Estudo realizado em 10 UTIs brasileiras, com pacientes clínicos e cirúrgicos, mostrou SMR de $1,66 .{ }^{8}$ Em nosso meio, em um estudo recente realizado na UTI do Hospital Universitário da UFSC, a SMR foi de 1,24 para amostra global, 1,29 para o grupo cirúrgico e 1,23 para o grupo clínico, o que denota uma boa relação entre as taxas prevista e observada. ${ }^{9}$ Observou-se ainda que no grupo de pacientes com índice APACHE II igual ou inferior a 30 pontos percentuais, havia uma melhor correlação entre a taxa de mortalidade real e a prevista; por outro lado, no grupo de pacientes com índice APACHE II superior 30 pontos percentuais, esta correlação não era tão evidente.

O sistema APACHE II mostrou-se um índice preditivo adequado de mortalidade em pacientes cirúrgicos que necessitaram de cuidados intensivos por um período superior a 24 horas, embora subestime a mortalidade em todas as faixas de variações dos índices alcançados.

A SMR foi de 1,55 para amostra global, 1,36 para o grupo com índice igual ou inferior a 30 pontos percentuais e para o grupo com índice APACHE II, superior a 30 pontos percentuais 1,62 , o que denota uma boa correlação entre as taxas prevista e observada na amostra global, e, principalmente no grupo com índice APACHE II, igual ou inferior a 30 pontos percentuais. Por outro lado, no grupo com índice APACHE II, superior a 30 pontos percentuais, esta relação não foi tão evidente.

\begin{abstract}
In the last fifteen years, a series of studies developing a prognostic scoring system for the evaluation of critically ill patients has been target. This project developed at the Hospital Regional de São José Dr. Homero de Miranda Gomes' Intensive Care Unit evalvetd the APACHE II scoring system's applicability and accuracy to surgical patients of this unit. All the surgical patients requiring intensive care for a period longer than 24 hours between January and July of 1996, were prospectively studied $(n=84)$. The average age of the group was $52.7 \pm 19.8$ range between 13 and 86 years. Sixty one patients $(72.62 \%)$ were male and $23(27.38 \%)$ female. Fifty-six patients $(66.67 \%)$ were submitted to emergency surgery and 28 patients (33.33\%) to elective surgery. Thirthy-two patients (38.09\%) died in the hospital. The predictive mortality of APACHE II scoring system was $24.6 \%$, with a Standardized Mortality Rate (SMR) of 1.55. The SMR to the 30 or less APACHE II points patients was 1.36, while greater than 30 was 1.62. The APACHE II score for the group was the 17.16 ( \pm 9.4 ). The APACHE II scoring system effectively predicted mortality for this group, mainly among the 30 points or less APACHE II point group.
\end{abstract}

Key Words: APACHE II; Predictive outcome.

\section{REFERÊNCIAS}

1. Cullen DJ, Civeta JM, Briggs BA, et al - Therapeutic intervention scoring system: A metod for quantitative comparison of patient care. Crit Care Med 1974;2:57.

2. Champion HR, Sacco WJ, Carnazzo AJ, et al - Trauma Score. Crit Care Med 1981;9:672.

3. Knaus WA, Zimmerman JE, Wagner DP, et al - APACHE - Acute physiology and chronic health evaluation: A physiologically based, classification system. Crit Care Med 1981;9:591-97.

4. Knaus WA, Draper EA, Wagner DP, et al - APACHE II: A severity of disease classification system. Crit Care Med 1985;13:818-29.

5. Knaus WA, Wagner DP, Bastos PG, et al - The APACHE III prognostic system. Risk prediction of hospital mortality for critically ill hospitalized adults. Chest 1991;100:1.619-36.

6. Terzi RGG - Índices prognósticos em medicina intensiva. Rev Bras Ter Intens 1993;5(04):101-06.

7. Rowan KM, Kerr JH, 'Major E, et al - Intensive care society's APACHE II study in Britain and Ireland - I: Variations in case mix of a adult admission to general intensive care units and impact on outcome. BMJ 1993;307:972-77.

8. Bastos PG, Sun X, Knaus WA, et al - Application of APACHE scoring system in brazilian (Br) ICUs. Crit Care Med 1992;20(4) Suppl:S79.

9. Silva E, et al - Sensibilidade e especificidade do sistema APACHE II para grupos distintos de pacientes. Rev Bras Terap Intens 1995,7(4): 11-15.

10. Lee KA, et al - Acute physiology and chronic health evaluation(APACHE II) scoring in the medical intensive care unit national university, Singapore. Singapore Med J 1993;34:41-44.

11. Chen FG, et al - Validation of APACHE II score in a surgical intensive care unit. Singapore Med J 1993;34:322-24.

\section{ENDEREÇO PARA CORRESPONDÊNCIA}

Dr. Waldiere Machado Gonçalves

Rua Delminda Silveira, 729/301

88025-500 - Florianópolis - SC 